\title{
Anaplastic Carcinoma Thyroid in a Young Child- an Extremely Rare Occurrence
}

\author{
Suresh Chandra Sharma ${ }^{1}$, Pirabu Sakthivel ${ }^{1, *}$, Sarath Raveendran ${ }^{1}$, Chirom Amit Singh ${ }^{1}$, Tripti Nakra ${ }^{2}$, \\ Shipra Agarwal²
}

\section{ABSTRACT}

Anaplastic thyroid carcinoma (ATC), one of the most aggressive malignancies, is extremely rare in childhood. We present a case of 5-yearold child who presented with rapidly progressing thyroid swelling and stridor, for which she underwent emergency tracheostomy and biopsy. Histopathological features were suggestive of ATC and the patient died within two months after diagnosis. ATC, though very rare in childhood, should be kept in the differential diagnoses of rapidly enlarging neck masses in children. To the best of our knowledge, this is the youngest case of ATC reported in literature.

\section{KEYWORDS}

thyroid; pediatric thyroid cancer; anaplastic carcinoma

\section{AUTHOR AFFILIATIONS}

${ }^{1}$ Department of Otorhinolaryngology \& Head and Neck Surgery, All India Institute of Medical Sciences, New Delhi, India

2 Department of Pathology, All India Institute of Medical Sciences, New Delhi, India

* Corresponding author: Room no 4057, ENT office, Fourth floor, Department of ENT \& Head and Neck surgery, All India Institute of Medical Sciences, New Delhi-110029, India; e- mail: pirabusakthivel@gmail.com

Received: 26 August 2018

Accepted: 12 November 2018

Published online: 22 January 2019

Acta Medica (Hradec Králové) 2018; 61(4): 150-152

https://doi.org/10.14712/18059694.2018.134

(c) 2018 The Authors. This is an open-access article distributed under the terms of the Creative Commons Attribution License (http://creativecommons.org/licenses/by/4.0), which permits unrestricted use, distribution, and reproduction in any medium, provided the original author and source are credited. 


\section{INTRODUCTION}

Pediatric thyroid malignancy is an uncommon diagnosis with most cases presenting in the late teens (1). Children less than 5 years account for less than $0.5 \%$ of the pediatric thyroid carcinomas, with more than $97 \%$ being differentiated cancers-papillary, follicular and medullary carcinomas in the decreasing order of frequency. Anaplastic thyroid carcinomas (ATC) are extremely rare in children with very few cases reported in the literature. Due to its aggressive course, mortality rate is more than $95 \%$. Most cases present with lymph node and systemic metastasis at presentation. There is no standard consensus in the treatment protocol for ATC $n$ children (2). To the best of our knowledge, this is the youngest case of ATC reported in literature till date.

\section{CASE REPORT}

A 5-year-old female child presented with a rapidly progressive midline neck swelling over two months duration. There was associated pain and the child presented to emergency with stridor. On examination, thyroid swelling of size approximately $6 \times 5 \mathrm{~cm}$ was seen with stretched out and shiny

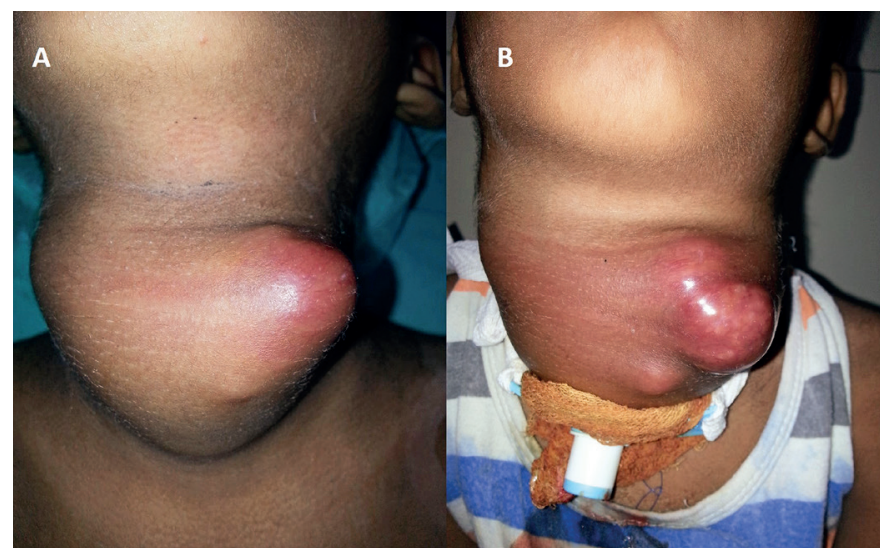

Fig. 1 A) Clinical picture before tracheostomy; B) After 5 days showing rapid growth and impending skin ulceration.

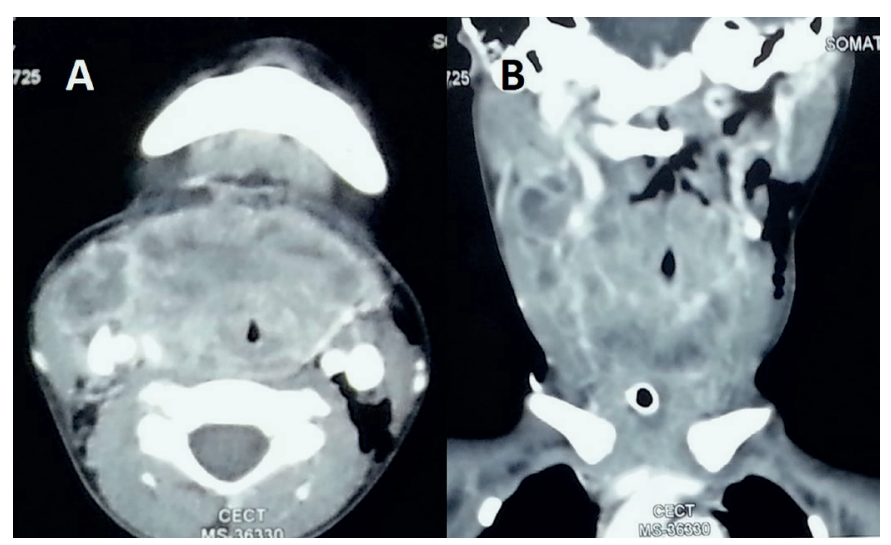

Fig. 3 CECT scan axial (A) and coronal cuts (B) showing heterogeneously enhancing soft tissue lesion is entirely replacing the thyroid gland, encasing the trachea with gross luminal narrowing and metastatic neck nodes. skin (Figure 1). The swelling was firm, tender and warm with restricted movement during deglutition. Child was in severe stridor, sitting in tripod posture due to bilateral cord palsy. There was no cervical lymphadenopathy. $X$ ray soft tissue neck revealed a large soft tissue mass in the anterior aspect of neck with marked tracheal luminal narrowing at the level of epiglottis (Figure 2). Emergency tracheostomy was performed followed by a biopsy from the thyroid mass, Contrast Enhanced Computed Tomography (CECT) scan showed a heterogeneously enhancing soft tissue lesion along with necrosis, entirely replacing the thyroid gland, and encasing the trachea with significant luminal narrowing. Enlarged lymph nodes with similar appearance as the thyroid mass were seen in the right level II and III stations (Figure 3). Clinical differential diagnoses included carcinoma, lymphoma, germ cell tumor and sarcoma. Serum calcitonin, thyroglobulin, AFP, beta HCG and LDH levels were all normal. There was no history of radiation exposure or family history of any thyroid cancer. Histopathology revealed sheets of atypical epithelial looking cells replacing the thyroid parenchyma with areas of necrosis. The tumor cells were large, polygonal with pleomorphic nuclei and prominent nucleoli along with numerous apoptotic bodies and mitotic figures. Immunohistochemically, the tumor cells were positive for EMA [strong] and cytokeratin

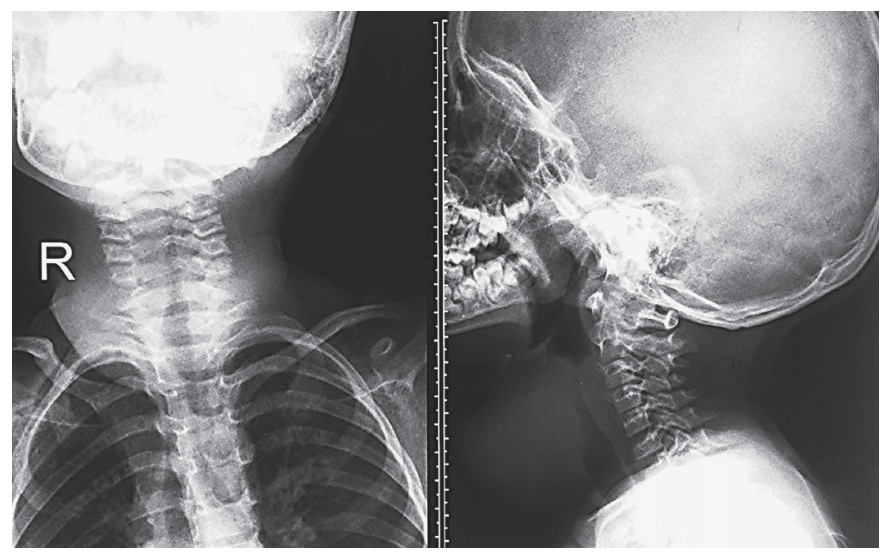

Fig. $2 \mathrm{X}$ rays showing large soft tissue mass in the anterior aspect of neck with marked tracheal luminal narrowing.

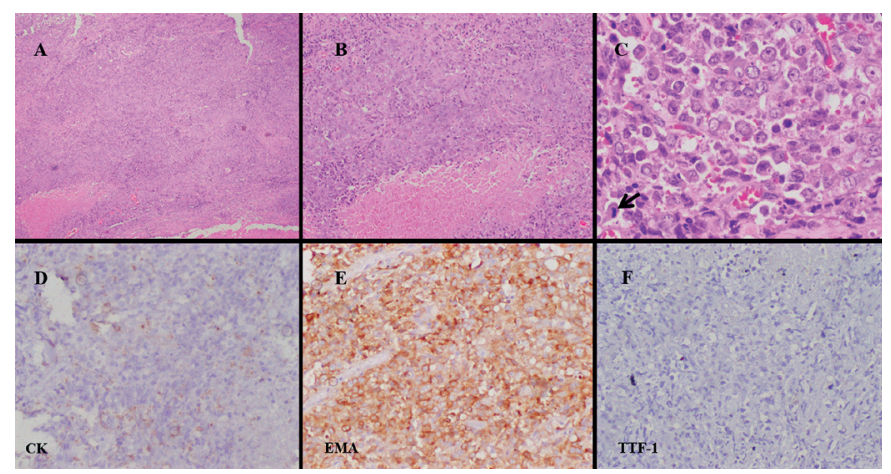

Fig. 4 (A), (B): 4×, 10× H\&E. Undifferentiated Carcinoma, sheets of atypical epithelial looking cells replacing the thyroid parenchyma. Areas of necrosis are evident. (C) The tumor cells are large, polygonal with pleomorphic nuclei and prominent nucleoli along with numerous apoptotic bodies and mitotic figures (arrow), 40x, $\mathrm{H} \& \mathrm{E}$. The tumor cells are focally immunopositive for pancytokeratin (D), diffusely immunopositive for Epithelial Membrane Antigen (E) and immunonegative for TTF-1 (F). 
[focal], confirming their epithelial nature, while were negative for LCA, EBV-LMP1, CD 3, CD 20, CD 30, ALK, CD 5, PLAP, CD117, TTF1 and thyroglobulin (Figure4). Overall features were suggestive of an undifferentiated ATC.

Due to extensive local disease, the child was planned for palliative chemotherapy and was started on docetaxel and doxorubicin. However, after 1st cycle of chemotherapy she succumbed to death due to disease progression.

\section{DISCUSSION}

Midline neck swellings in children have wide differentials like thyroid mass, dermoid cyst, lymph nodes, vascular malformations, thymic mass, etc. (3). Careful history about the onset of the symptoms, its course and past history of any radiation should be obtained from caretakers. Clinical examination can narrow down the provisional diagnosis, but radiology is usually warranted in young children as accurate clinical examination can often be difficult. However, thyroid origin was apparent on examination in our case.

ATC incidence typically peaks at the 6-7th decade of life with mean age at diagnosis of 55-65 years (4). Mon et al described a case of anaplastic carcinoma thyroid in a 12 year old female child which is the youngest reported case in the literature till date (5).The patients usually have an advanced disease at presentation and may have symptoms like hoarseness, stridor, dysphagia, or skin ulceration depending on the extent of invasion, and superior vena cava syndrome due to thoracic inlet obstruction. Evaluation for local and systemic metastasis should done as there is a $50 \%$ chance of lymph node metastasis. Lungs, bone and brain metastasis have been reported in decreasing order of frequency (6).

ATC usually arises by dedifferentiation of differentiated thyroid cancer, but de-novo development from multinodular goiter has also been documented (4). It has been shown that they harbor the same BRAF mutation when they develop from BRAF mutated papillary carcinoma. More than $20 \%$ of anaplastic carcinomas have differentiated component in histological sections, typically in patients having a history of long standing thyroid nodule with recent rapid increase in size and symptoms (7). Elevated serum thyroglobulin can be a marker of the differentiated component within the tumor rather than the anaplastic variant itself (8). Our child had normal serum thyroglobulin levels, and neither had any history of longstanding thyroid swelling nor any differentiated component in the biopsy, raising the possibility of de-novo genesis of anaplasia rather than dedifferentiation.

A CECT of neck with chest should be a minimum screening protocol in anaplastic carcinoma cases. Lee et al found that tumor necrosis was the most valuable parameter in CECT for differentiating anaplastic carcinomas from other thyroid masses (9). Other imaging modalities include contrast enhanced magnetic resonance imaging (CEMRI) and FDG-positron enhanced tomography. CEMRI has been shown to have superior sensitivity and specificity compared to CECT in detecting local extension to soft tissue, esophagus and recurrent laryngeal nerve(10). However, FDG-PET is a valuable tool to differentiate ATC metastases, which are highly FDG avid, from more indolent metastases typically observed in differentiated thyroid cancers (11).

Treatment options in ATC remain controversial. Due to the aggressive course of this condition, median survival is less than 5 months and mortality is more than $95 \%(2,12)$. Aggressive surgery followed by chemotherapy and external beam radiotherapy and have shown to increase the survival in loco regionally confined diseases (13). Neo-adjuvant therapy is also reported to de-bulk the disease which makes resection possible. Pudney et al. reported a median survival of 13 months for five patients with stage IVB ATC treated with either radiation therapy concurrent with doxorubicin, or induction with docetaxel, doxorubicin and cyclophosphamide (14).

\section{CONCLUSION}

ATC though very rare in childhood, should be kept in the differential diagnosis of rapidly enlarging neck masses in children. Early diagnosis and prompt therapy, especially in resectable cases, will help to improve the prognosis of this aggressive tumor.

\section{CONSENT}

Written informed consent was obtained from the parents for publication of this case report and accompanying images.

\section{REFERENCES}

1. Dermody S, Walls A, Harley EH Jr. Pediatric thyroid cancer: An update from the SEER database 2007-2012. Int J Pediatr Otorhinolaryngol 2016; 89: 121-6.

2. Hogan AR, Zhuge Y, Perez EA, Koniaris LG, Lew JI, Sola JE. Pediatric thyroid carcinoma: incidence and outcomes in 1753 patients. J Surg Res 2009; 156: 167-72.

3. Meier JD, Grimmer JF. Evaluation and management of neck masses in children. Am Fam Physician 2014; 89: 353-8.

4. Chiacchio S, Lorenzoni A, Boni G, Rubello D, Elisei R, Mariani G. Anaplastic thyroid cancer: prevalence, diagnosis and treatment. Minerva Endocrinol 2008; 33: 341-57.

5. Mon R, Newlon J. Anaplastic carcinoma of the thyroid in a 12-year old girl. J Ped Surg Case Reports 2015; 3: 404-6.

6. Keutgen XM, Sadowski SM, Kebebew E. Management of anaplastic thyroid cancer. Gland Surg 2015; 4: 44-51.

7. Moore JH Jr, Bacharach B, Choi HY. Anaplastic transformation of metastatic follicular carcinoma of the thyroid. J Surg Oncol 1985; 29: 216-21.

8. Herle AJ, Uller RP. Elevated serum thyroglobulin. A marker of metastases in differentiated thyroid carcinomas. J Clin Invest 1975; 56: 272-7.

9. Lee JW, Yoon DY, Choi CS, Chang SK, Yun EJ, Seo YL, Rho YS, Cho SJ, Kim $\mathrm{KH}$. Anaplastic thyroid carcinoma: computed tomographic differentiation from other thyroid masses. Acta Radiol 2008; 49: 321-7.

10. Hoang JK, Branstetter BF 4th, Gafton AR, Lee WK, Glastonbury CM. Imaging of thyroid carcinoma with $C T$ and MRI: approaches to common scenarios. Cancer Imaging 2013; 13: 128-39.

11. Marcus C, Whitworth PW, Surasi DS, Pai SI, Subramaniam RM. PET/CT in the management of thyroid cancers. AJR Am J Roentgenol 2014; 202: 1316-29.

12. Perri F, Lorenzo GD, Scarpati GD, Buonerba C. Anaplastic thyroid carcinoma: A comprehensive review of current and future therapeutic options. World J Clin Oncol 2011; 2: 150-7.

13. Käsmann L, Bolm L, Janssen S, Rades D. Prognostic Factors for Survival in Patients Treated with Multimodal Therapy for Anaplastic Thyroid Cancer. Anticancer Res 2016; 36: 4697-700.

14. Pudney D, Lau H, Ruether JD, Falck V. Clinical experience of the multimodality management of anaplastic thyroid cancer and literature review. Thyroid 2007; 17: 1243-50. 